\title{
Sharp Conditions for the Oscillation of Delay Difference Equations
}

\author{
G. LADAS \\ Department of Mathematics \\ The University of Rhode Island \\ Kingston, R.I. 02881, U.S.A. \\ Ch. G. PHILOS and Y.G. SFICAS \\ Department of Mathematics \\ University of Ioannina \\ Ioannina 45110, P.O. Box 1186 \\ Greece
}

\begin{abstract}
Suppose that $\left\{p_{n}\right\}$ is a nonnegative sequence of real numbers and let $k$ be a positive integer. We prove that

$$
\liminf _{n \rightarrow \infty}\left[\frac{1}{k} \sum_{i=n-k}^{n-1} p_{i}\right]>\frac{k^{k}}{(k+1)^{k+1}}
$$

is a sufficient condition for the oscillation of all solutions of the delay difference equation

$$
A_{n+1}-A_{n}+p_{n} A_{n-k}=0, \quad n=0,1,2, \ldots .
$$

This result is sharp in that the lower bound $k^{k} /(k+1)^{k+1}$ in the condition cannot be improved. Some results on difference inequalities and the existence of positive solutions are also presented.
\end{abstract}

Key words: Oscillations, Difference equations, Positive solutions, Difference inequalities.

AMS Subject Classification: Primary - 39A10. 


\section{INTRODUCTION AND PRELIMINARIES}

Recently there has been some activity concerning the oscillation of all solutions of the delay difference equation

$$
A_{n+1}-A_{n}+p_{n} A_{n-k}=0, \quad n=0,1,2, \ldots
$$

where $\left\{p_{n}\right\}$ is a sequence of nonnegative real numbers and $k$ is a positive integer. See, for example, [1]-[3] and the references cited therein. Throughout this paper, the sequence $\left\{p_{n}\right\}$ is supposed to be defined for $n \geq 0$.

By a solution of Eq. (1) we mean a sequence $\left\{A_{n}\right\}$ which is defined for $n \geq-k$ and which satisfies Eq. (1) for $n \geq 0$. A solution $\left\{A_{n}\right\}$ of Eq. (1) is said to be oscillatory if the terms $A_{n}$ of the sequence are not eventually positive or eventually negative. Otherwise, the solution is called nonoscillatory.

Our aim in Section 2 is to establish the following result.

Theorem 1. Suppose that $\left\{p_{n}\right\}$ is a nonnegative sequence of real numbers and let $k$ be a positive integer. Then

$$
\liminf _{n \rightarrow \infty}\left[\frac{1}{k} \sum_{i=n-k}^{n-1} p_{i}\right]>\frac{k^{k}}{(k+1)^{k+1}}
$$

is a sufficient condition for every solution of Eq. (1) to be oscillatory.

This theorem is sharp in that the lower bound $k^{k} /(k+1)^{k+1}$ cannot be improved. Moreover, when

$$
p_{n}=p \in(0, \infty) \text { for } n=0,1,2, \ldots
$$

condition (2) reduces to

$$
p>\frac{k^{k}}{(k+1)^{k+1}}
$$

which is a necessary and sufficient condition for the oscillation of all solutions of the difference equation

$$
A_{n+1}-A_{n}+p A_{n-k}=0, \quad n=0,1,2, \ldots
$$

For a proof of this result see [3]. If

$$
\liminf _{n \rightarrow \infty} p_{n}>\frac{k^{k}}{(k+1)^{k+1}}
$$


then it follows from [2] that every solution of Eq. (1) oscillates. Clearly (2) is a substantial improvement over (5), replacing the $p_{n}$ of (5) by the arithmetic mean of the terms $p_{n-k}, \ldots, p_{n-1}$ in (2).

Theorem 1 should be looked upon as a discrete analogue of the wellknown theorem about the oscillation of the delay differential equation,

$$
\dot{x}(t)+p(t) x(t-\tau)=0, \quad t \geq t_{0}
$$

where

$$
p \in C\left[\left[t_{0}, \infty\right),[0, \infty)\right] \text { and } \tau \in(0, \infty),
$$

which states that

$$
\liminf _{t \rightarrow \infty} \int_{t-\tau}^{t} p(s) d s>\frac{1}{e}
$$

is a sufficient condition for the oscillation of all solutions of Eq. (6). See [4]. We should also remark here that it is the proof of this latter theorem (see Theorem 2.1.1 in [4]) which we used as our guide in arriving at the statement and the proof of Theorem 1 . One should notice that condition (2) can be written in the form

$$
\liminf _{n \rightarrow \infty} \sum_{i=n-k}^{n-1} p_{i}>\left(\frac{k}{k+1}\right)^{k+1}
$$

and that

$$
\lim _{k \rightarrow \infty}\left(\frac{k}{k+1}\right)^{k+1}=\lim _{k \rightarrow \infty}\left[\frac{1}{\left(1+\frac{1}{k}\right)^{k}} \cdot \frac{1}{1+\frac{1}{k}}\right]=\frac{1}{e} .
$$

Finally we should mention that another sufficient condition for the oscillation of all solutions of Eq. (1) is

$$
\limsup _{n \rightarrow \infty} \sum_{i=n-k}^{n} p_{i}>1
$$

which was given by Erbe and Zhang in [1].

In Section 3 we present some results about difference inequalities. In particular we prove that, under appropriate hypotheses, if the difference inequality

$$
x_{n+1}-x_{n}+p_{n} x_{n-k} \leq 0, \quad n=0,1,2, \ldots
$$

has a positive solution so does Eq. (1). Finally, we utilize this result to give a "sharp" sufficient condition for the existence of a positive solution of Eq. (1). 


\section{PROOF OF THEOREM 1}

Assume, for the sake of contradiction, that Eq. (1) has a nonoscillatory solution $\left\{A_{n}\right\}$. As the opposite of a solution of Eq. (1) is also a solution, we may (and do) assume that $\left\{A_{n}\right\}$ is eventually positive. Then eventually

$$
A_{n+1}-A_{n}=-p_{n} A_{n-k} \leq 0,
$$

and so $\left\{A_{n}\right\}$ is an eventually decreasing sequence of positive numbers. It follows from Eq. (1) that eventually

$$
A_{n+1}-A_{n}+p_{n} A_{n} \leq 0
$$

or

$$
p_{n} \leq 1-\frac{A_{n+1}}{A_{n}}
$$

and so eventually,

$$
\frac{1}{k} \sum_{i=n-k}^{n-1} p_{i} \leq \frac{1}{k} \sum_{i=n-k}^{n-1}\left(1-\frac{A_{i+1}}{A_{i}}\right) .
$$

Set

$$
\alpha=\frac{k^{k}}{(k+1)^{k+1}} .
$$

Then, from (2), it is clear that we can choose a constant $\beta$ such that, for $n$ sufficiently large,

$$
\alpha<\beta \leq \frac{1}{k} \sum_{i=n-k}^{n-1} p_{i}
$$

Thus, in view of (7),

$$
\beta \leq \frac{1}{k} \sum_{i=n-k}^{n-1}\left(1-\frac{A_{i+1}}{A_{i}}\right) \quad \text { for all large } n .
$$

By using (10) and the well-known inequality between the arithmetic and geometric means we find that for $n$ sufficiently large,

$$
\begin{aligned}
\beta & \leq \frac{1}{k} \sum_{i=n-k}^{n-1}\left(1-\frac{A_{i+1}}{A_{i}}\right)=1-\frac{1}{k} \sum_{i=n-k}^{n-1} \frac{A_{i+1}}{A_{i}} \\
& \leq 1-\left(\prod_{i=n-k}^{n-1} \frac{A_{i+1}}{A_{i}}\right)^{1 / k}=1-\left(\frac{A_{n}}{A_{n-k}}\right)^{1 / k},
\end{aligned}
$$


that is,

$$
\left(\frac{A_{n}}{A_{n-k}}\right)^{1 / k} \leq 1-\beta \text { for all large } n .
$$

In particular, this implies that $0<\beta<1$.

Now observe that

$$
\max _{0 \leq \lambda \leq 1}\left[(1-\lambda) \lambda^{1 / k}\right]=\frac{k}{(k+1)^{1+\frac{1}{k}}}=\alpha^{1 / k}
$$

where $\alpha$ is the positive constant defined by (8). Therefore

$$
1-\lambda \leq \alpha^{1 / k} \lambda^{-1 / k} \text { for } 0<\lambda \leq 1
$$

and (11) yields

$$
\frac{\beta}{\alpha} A_{n} \leq A_{n-k} \text { for all large } n .
$$

By using (12) in Eq. (1) and then by repeating the above arguments we find that

$$
\left(\frac{\beta}{\alpha}\right)^{2} A_{n} \leq A_{n-k} \text { for all large } n
$$

and, by induction, for every $m=1,2, \ldots$ there exists an integer $n_{m}$ such that for $n \geq N_{m}$,

$$
\left(\frac{\beta}{\alpha}\right)^{m} A_{n} \leq A_{n-k}
$$

Next observe that because of (9), for $n$ sufficiently large,

$$
\sum_{i=n-k}^{n} p_{i} \geq \sum_{i=n-k}^{n-1} p_{i} \geq k \beta .
$$

Hence, for $n$ sufficiently large,

$$
\sum_{i=n-k}^{n} p_{i} \geq M
$$

where $M=k \beta>0$. Choose $m$ such that

$$
\left(\frac{\beta}{\alpha}\right)^{m}>\left(\frac{2}{M}\right)^{2} \text {. }
$$

This is possible because from (9), $\beta>\alpha$. Then for $n$ sufficiently large, say for $n \geq n_{0},(13)$ is satisfied for the specific $m$ which was chosen in (15), also 
(9) and (14) hold, and $\left\{A_{n}\right\}$ is decreasing for $n \geq n_{0}$. Now in view of (14) and for $n \geq n_{0}+k$, there exists an integer $n^{*}$ with $n-k \leq n^{*} \leq n$ such that

$$
\sum_{i=n-k}^{n^{*}} p_{i} \geq \frac{M}{2} \quad \text { and } \quad \sum_{i=n^{*}}^{n} p_{i} \leq \frac{M}{2}
$$

From Eq. (1) and the decreasing nature of $\left\{A_{n}\right\}$, we have

$$
\begin{aligned}
A_{n^{*}-1}-A_{n-k} & =\sum_{i=n-k}^{n^{*}}\left(A_{i+1}-A_{i}\right) \\
& =-\sum_{i=n-k}^{n^{*}} p_{i} A_{i-k} \\
& \leq-\left(\sum_{i=n-k}^{n^{*}} p_{i}\right) A_{n^{*}-k} \\
& \leq-\frac{M}{2} A_{n^{*}-k}
\end{aligned}
$$

Hence,

$$
\frac{M}{2} A_{n \cdot-k} \leq A_{n-k}
$$

Similarly,

$$
\begin{aligned}
A_{n+1}-A_{n^{*}} & =\sum_{i=n^{*}}^{n}\left(A_{i+1}-A_{i}\right) \\
& =-\sum_{i=n^{*}}^{n} p_{i} A_{i-k} \\
& \leq-\left(\sum_{i=n^{*}}^{n} p_{i}\right) A_{n-k} \\
& \leq-\frac{M}{2} A_{n-k}
\end{aligned}
$$

and so

$$
\frac{M}{2} A_{n-k} \leq A_{n}
$$

From (16) and (17) we find

$$
\left(\frac{M}{2}\right)^{2} A_{n^{*}-k} \leq A_{n^{*}}
$$


which in view of (13) yields

$$
\left(\frac{\beta}{\alpha}\right)^{m} \leq \frac{A_{n^{*}-k}}{A_{n^{*}}} \leq\left(\frac{2}{M}\right)^{2} .
$$

This contradicts (15) and so the proof of the theorem is complete.

\section{DIFFERENCE INEQUALITIES}

A slight modification in the proof of Theorem 1 leads to the following result about the difference inequalities,

$$
x_{n+1}-x_{n}+p_{n} x_{n-k} \leq 0, \quad n=0,1,2, \ldots
$$

and

$$
y_{n+1}-y_{n}+p_{n} y_{n-k} \geq 0, \quad n=0,1,2, \ldots,
$$

where $\left\{p_{n}\right\}$ is a sequence of nonnegative real numbers and $k$ is a positive integer.

By a solution of (18) we mean a sequence $\left\{x_{n}\right\}$ which is defined for $n \geq-k$ and which satisfies (18) for $n \geq 0$. Solutions of (19) are defined in a similar manner.

Theorem 2. Assume that $\left\{p_{n}\right\}$ is a nonnegative sequence of real numbers and let $k$ be a positive integer. Suppose that (2) holds. Then (18) cannot have eventually positive solutions and (19) cannot have eventually negative solutions.

The following result shows that, under appropriate hypotheses, if (18) has a positive solution so does Eq. (1).

Theorem 3. Let $k$ be a positive integer and let $\left\{p_{n}\right\}$ be a sequence of nonnegative real numbers such that

$$
\sum_{j=0}^{k-1} p_{n+j}>0 \text { for } n \geq 0 .
$$

Assume that $\left\{x_{n}\right\}$ is a solution of (18) such that

$$
x_{n}>0 \text { for } n \geq-k .
$$

Then Eq. (1) has a solution $\left\{A_{n}\right\}$ such that

$$
0<A_{n} \leq x_{n} \text { for } n \geq-k \text { and } \lim _{n \rightarrow \infty} A_{n}=0 .
$$


Proof. For $\tilde{n} \geq n \geq 0$ we have

$$
x_{\tilde{n}+1}-x_{n}+\sum_{i=n}^{\tilde{n}} p_{i} x_{i-k} \leq 0
$$

and so

$$
\sum_{i=n}^{\infty} p_{i} x_{i-k} \leq x_{n} \text { for } n=0,1,2, \ldots
$$

Consider the space $S$ of all sequences $\left\{A_{n}\right\}$ for $n \geq-k$ which are such that

$$
A_{n}=x_{n} \text { for } \quad-k \leq n<0
$$

and

$$
0 \leq A_{n} \leq x_{n} \text { for } n \geq 0 .
$$

Define the operator $T$ on $S$ as follows. For every $A=\left\{A_{n}\right\} \in S$, set $T A=B=\left\{B_{n}\right\}$ where

$$
B_{n}=x_{n} \text { for } \quad-k \leq n<0
$$

and

$$
B_{n}=\sum_{i=n}^{\infty} p_{i} A_{i-k} \quad \text { for } \quad n \geq 0 .
$$

It follows from (22) that

$$
B_{n} \leq \sum_{i=n}^{\infty} p_{i} x_{i-k} \leq x_{n} \text { for } n \geq 0
$$

and so $T$ is well-defined and $T: S \rightarrow S$.

If $A^{1}=\left\{A_{n}^{1}\right\}$ and $A^{2}=\left\{A_{n}^{2}\right\}$ are two sequences in $S$, we will say that $A^{1} \leq A^{2}$ if and only if $A_{n}^{1} \leq A_{n}^{2}$ for $n \geq-k$. With this definition, the operator $T$ is monotonic in the sense that if $A^{1}, A^{2} \in S$ with $A^{1} \leq A^{2}$ then $T A^{1} \leq T A^{2}$.

Next, we define the sequence $\left\{A^{r}\right\}$ for $r=0,1,2, \ldots$ of points $A^{r} \in S$ in the following way:

$$
A^{0}=\left\{x_{n}\right\}
$$

and

$$
A^{r+1}=T A^{r} \quad \text { for } \quad r=0,1,2, \ldots .
$$

It follows by induction that

$$
\cdots \leq A^{r+1} \leq A^{r} \leq \cdots \leq A^{1} \leq A^{0} .
$$


Set

$$
A^{r}=\left\{A_{n}^{r}\right\} \text { for } r=0,1,2, \ldots \text { and } A_{n}=\lim _{r \rightarrow \infty} A_{n}^{r} .
$$

Then we can see that, for every $n \geq 0$,

$$
A_{n}=\sum_{i=n}^{\infty} p_{i} A_{i-k}
$$

and so

$$
A_{n+1}-A_{n}=-p_{n} A_{n-k} \text { for } n=0,1,2, \ldots \text {, }
$$

that is, $\left\{A_{n}\right\}$ is a solution of Eq. (1). It is also clear that

$$
0 \leq A_{n} \leq x_{n} \text { for } n \geq-k \text { and } \lim _{n \rightarrow \infty} A_{n}=0 .
$$

Finally, we claim that $A_{n}>0$ for $n \geq-k$. Otherwise, there exists $n_{0} \geq 0$ such that

$$
A_{n}>0 \text { for } n=-k, \ldots, n_{0}-1 \text { and } A_{n_{0}}=0 .
$$

Then, by summing up both sides of (25) from $n=n_{0}$ to $n=n_{0}+k-1$ and by taking into account (20), we find

$$
\begin{aligned}
0 \leq A_{n_{0}+k} & =-\sum_{j=n_{0}}^{n_{0}+k-1} p_{j} A_{j-k}<0 \\
& \leq-\left(\min _{n_{0} \leq j \leq n_{0}+k-1} A_{j-k}\right) \sum_{j=n_{0}}^{n_{0}+k-1} p_{j} \\
& =-\left(\min _{n_{0}-k \leq j \leq n_{0}-1} A_{j}\right) \sum_{j=0}^{k-1} p_{n_{0}+j} \\
& <0 .
\end{aligned}
$$

This is a contradiction and the proof is complete.

The following corollary of Theorem 3 provides a sufficient condition for the existence of a positive solution of Eq. (1).

Corollary 1. Let $k$ be a positive integer and let $\left\{p_{n}\right\}$ be a sequence of nonnegative real numbers such that $(20)$ is satisfied. Assume that there exists a number $\gamma \in(0,1)$ such that

$$
p_{n}<\gamma \text { for } n=0,1,2, \ldots
$$


and

$$
\prod_{i=n-k}^{n-1}\left(1-\frac{1}{\gamma} \tilde{p}_{i}\right) \geq \gamma \text { for } n \geq 0
$$

where

$$
\tilde{p}_{n}= \begin{cases}p_{n} & \text { for } n \geq 0 \\ p_{0} & \text { for } n<0 .\end{cases}
$$

Then Eq. (1) has a solution $\left\{A_{n}\right\}$ which is positive for $n \geq-k$ and is such that

$$
\lim _{n \rightarrow \infty} A_{n}=0 \text {. }
$$

Proof. Set

$$
x_{n}=\prod_{i=-k-1}^{n-1}\left(1-\frac{1}{\gamma} \tilde{p}_{i}\right) \text { for } n \geq-k .
$$

Clearly, $x_{n}>0$ for $n \geq-k$ and, by Theorem 3 , it suffices to show that $\left\{x_{n}\right\}$ is a solution of the difference inequality (18). To this end, in view of (27) we have, for $n \geq 0$,

$$
\begin{aligned}
x_{n+1}-x_{n} & =\left[\left(1-\frac{1}{\gamma} \tilde{p}_{n}\right)-1\right] \prod_{i=-k-1}^{n-1}\left(1-\frac{1}{\gamma} \tilde{p}_{i}\right) \\
& =-\frac{1}{\gamma} p_{n}\left[\prod_{i=-k-1}^{n-k-1}\left(1-\frac{1}{\gamma} \tilde{p}_{i}\right)\right]\left[\prod_{i=n-k}^{n-1}\left(1-\frac{1}{\gamma} \tilde{p}_{i}\right)\right] \\
& =-p_{n} x_{n-k}\left[\frac{1}{\gamma} \prod_{i=n-k}^{n-1}\left(1-\frac{1}{\gamma} \tilde{p}_{i}\right)\right] \\
& \leq-p_{n} x_{n-k}
\end{aligned}
$$

and the proof is complete.

We can see that (27) is a "sharp" condition for the existence of a positive solution of Eq. (1) in the sense that when $p_{n}$ is a constant $p$ then (27) becomes

$$
\left(1-\frac{1}{\gamma} p\right)^{k} \geq \gamma
$$

or equivalently

$$
p \leq \gamma\left(1-\gamma^{1 / k}\right)
$$

But

$$
\max _{0 \leq \gamma \leq 1}\left[\gamma\left(1-\gamma^{1 / k}\right)\right]=\left[\gamma\left(1-\gamma^{1 / k}\right)\right]_{\gamma=(k / k+1)^{k}}=\frac{k^{k}}{(k+1)^{k+1}}
$$


Hence with $\gamma=\left(\frac{k}{k+1}\right)^{k},(27)$ is satisfied provided that

$$
p \leq \frac{k^{k}}{(k+1)^{k+1}}
$$

Note also that, in the case where

$$
p \leq \frac{k^{k}}{(k+1)^{k+1}}<\left(\frac{k}{k+1}\right)^{k}=\gamma,
$$

(26) is also satisfied. Now as we mentioned in the introduction of the paper, (3) is a necessary and sufficient condition for the oscillation of every solution of Eq. (4). Hence (29) is a necessary and sufficient condition for the existence of a positive solution of Eq. (4).

\section{REFERENCES}

[1] L.H. Erbe and B.G. Zhang, Oscillation of Discrete Analogues of Delay Equations, Proceedings of the International Conference on Theory and Applications of Differential Equations, March 21-25, 1988, Ohio University, to be published by Marcel Dekker, Inc.

[2] I. Györi and G. Ladas, Linearized Oscillations for Equations with Piecewise Constant Arguments, Differential and Integral Equations (to appear).

[3] G. Ladas, Oscillations of Equations with Piecewise Constant Mixed Arguments, Proceedings of the International Conference on Theory and Applications of Differential Equations, March 21-25, 1988, Ohio University, to be published by Marcel Dekker, Inc.

[4] G.S. Ladde, V. Lakshmikantham and B.G. Zhang, Oscillation Theory of Differential Equations With Deviating Arguments, Marcel Dekker, Inc., New York, 1987. 


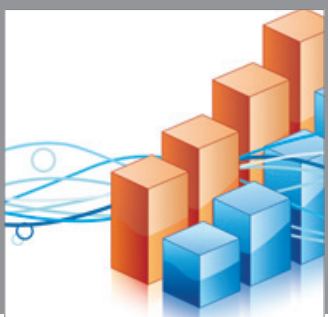

Advances in

Operations Research

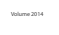

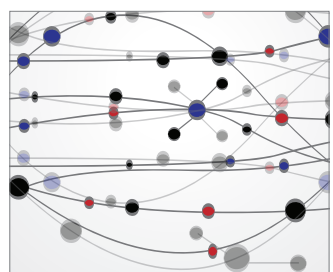

\section{The Scientific} World Journal
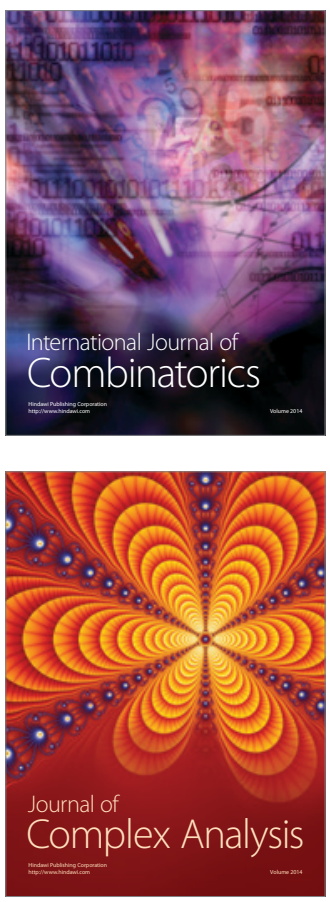

International Journal of

Mathematics and

Mathematical

Sciences
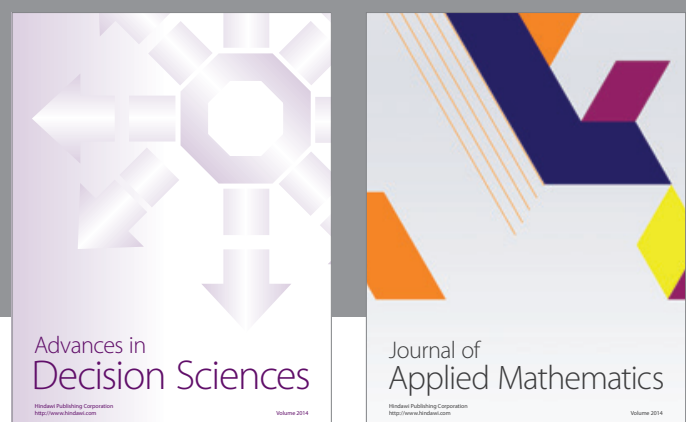

Journal of

Applied Mathematics
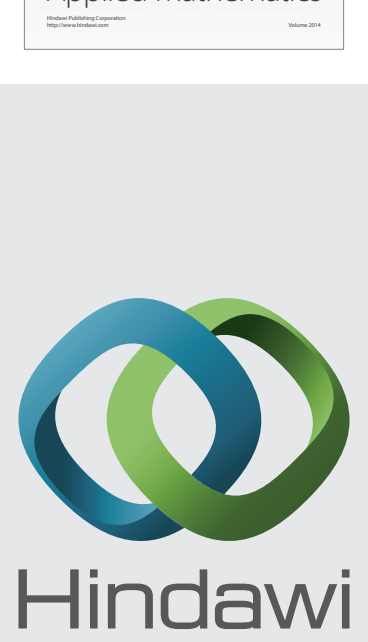

Submit your manuscripts at http://www.hindawi.com
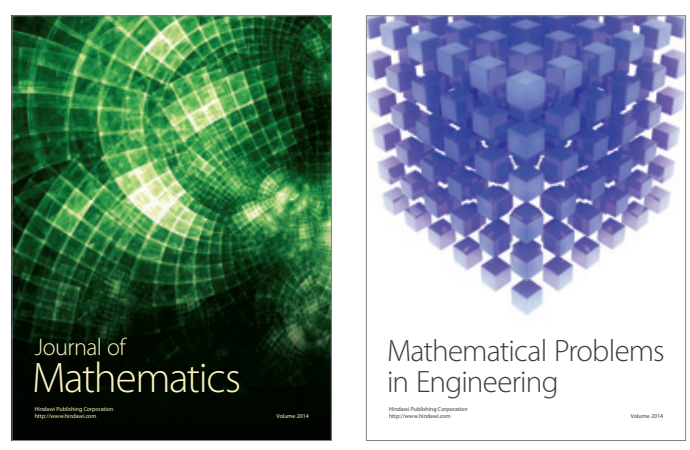

Mathematical Problems in Engineering
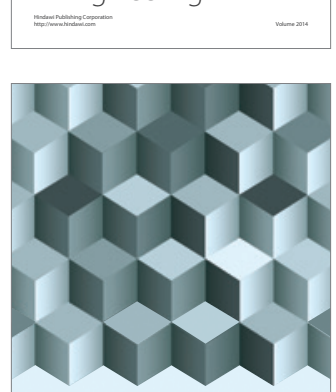

Journal of

Function Spaces
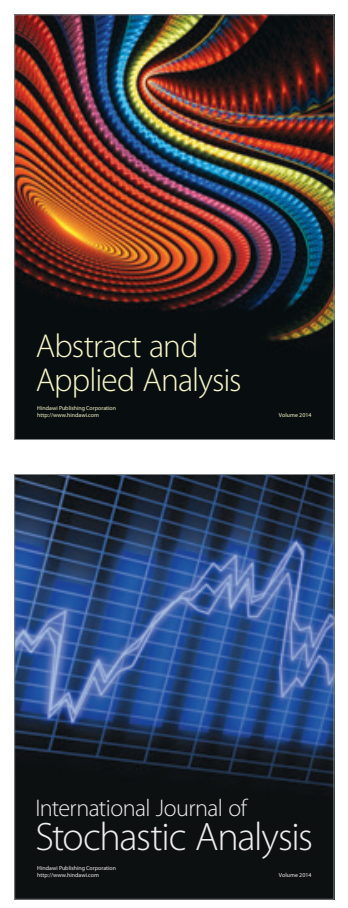

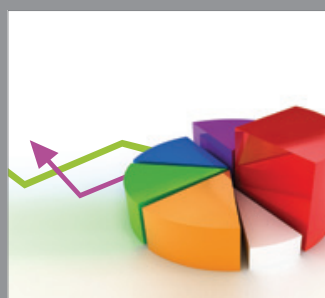

ournal of

Probability and Statistics

Promensencen
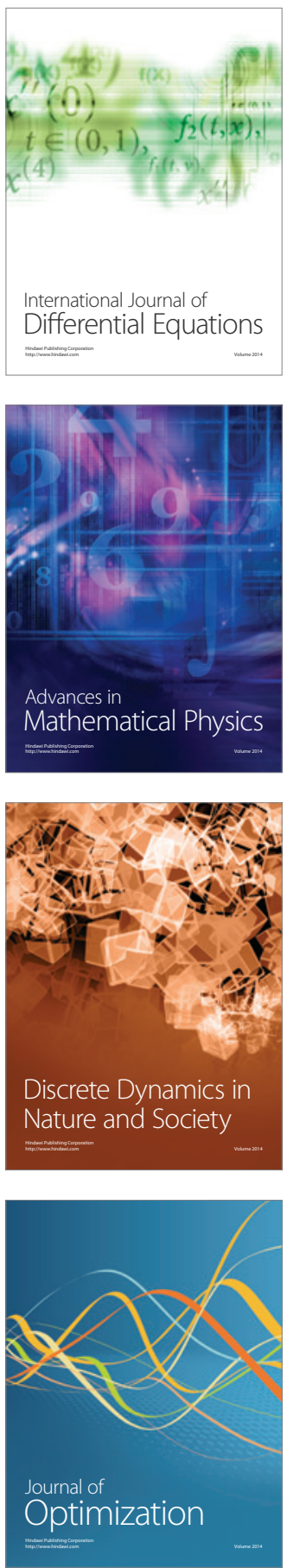